\title{
Toxic shock syndrome associated arthropathy. Staphylococcus aureus: A further triggering event in reactive arthritis?
}

\author{
D FOLEY-NOLAN, R J COUGHLAN, AND D SUGRUE
}

From the Department of Rheumatology and Rehabilitation, Mater Misericordiae Hospital, Dublin, Ireland

SUMmaRY An HLA-B27 positive 24 year old woman is described with a Staphylococcus aureus induced toxic shock syndrome precipitating a reactive arthritis.

Patients who are HLA-B27 positive are predisposed when they incubate certain organisms to develop reactive arthritis. To date enteric pathogens shigella, salmonella, yersinia, and campylobacter as well as sexually acquired chlamydia have been reported to precipitate this type of arthropathy. ${ }^{1}$ This report is of an HLA-B27 positive 24 year old woman with a Staphylococcus aureus induced toxic shock syndrome precipitating a reactive arthritis, which has been clinically active during 14 months' follow up.

This is the first report of an $S$ aureus associated toxic shock syndrome triggering a reactive arthritis in a genetically susceptible host.

\section{Case report}

A 24 year old woman presented with a 36 hour history of rigors, dry cough, arthralgias, myalgias, dizziness, and lethargy. She was menstruating at the time using tampons and gave a one day history of purulent vaginal discharge. On admission she complained of slight chest discomfort, though examination of her lungs and praecordium were normal. Vital signs showed a sinus tachycardia, a pyrexia of $39^{\circ} \mathrm{C}$, and a blood pressure of $100 / 60$ $\mathrm{mmHg}$. Other important physical findings were bilateral conjunctivitis, bilateral metatarsalgia, and a tender right temperomandibular joint. She also had painful limitation of movement of her right ankle, right knee, and left elbow with swelling of these joints.

Accepted for publication 4 August 1988.

Correspondence to Dr D Foley-Nolan, Department of Rheumatology and Rehabilitation, Mater Misericordiae Hospital, Dublin 7, Ireland.
Investigations showed a growth of $S$ aureus on a high vaginal swab and midstream urine, a mild $\left(10.5 \times 10^{9} \mathrm{~g} / \mathrm{l}\right)$ polymorpholeucocytosis, a sedimentation rate of $13 \mathrm{~mm} / \mathrm{h}$, and repeatedly negative blood cultures. Fluid from the right knee and left elbow was sterile. $x$ Rays of her chest, knees, ankles, wrists, and hands were normal. HLA typing showed a B27 haplotype. Later in the course of her arthritis she developed pain over her left sacroiliac joint. A bone scan showed no evidence of increased activity.

Within 72 hours of starting treatment with intravenous cloxacillin she became apyrexial and her acute illness settled over the following days. A week after stopping antibiotics she again developed painful stiff joints. At this time she was found to have a tender right parotid gland, a tender right temperomandibular joint, a flexion contracture of her left elbow with associated synovitis, and painful swollen restricted ankles, knees, and metatarsophalangeal joints. Sedimentation rate was raised at $29 \mathrm{~mm} / \mathrm{h}$ with a normal white cell count and temperature. Repeated aspirations of her joints showed no growth.

Over the ensuing 14 month period she has had recurrent episodes of synovitis in both knees despite repeated aspiration and several intra-articular corticosteroid injections. Blood count, erythrocyte sedimentation rate, and $\mathrm{C}$ reactive protein concentrations have been normal over this period.

\section{Discussion}

The only previous report of a patient with the toxic shock syndrome and associated arthritis was a 
15 year old girl who developed a wrist synovitis associated with rash and fever and whose symptoms, including her synovitis, settled within 24 hours of starting treatment with cloxacillin. ${ }^{2}$ Other reports have mentioned arthralgia as a feature of the toxic shock syndrome. ${ }^{34}$

The close association between HLA-B27 and reactive arthritis implies that this genetic haplotype is important in the ensuing reactive arthritis. ${ }^{5}$ Our patient, with the HLA-B27 haplotype, had a chronic arthritis following the toxic shock syndrome. This arthritis was asymmetrical and involved the large joints, features characteristic of reactive arthritis. Is this arthropathy a previously undescribed feature of toxic shock syndrome or can $S$ aureus of certain phage types trigger reactive arthritis in a genetically susceptible host? $S$ aureus has not previously been? stated to trigger a reactive arthritis and we propose $\vec{F}$ that it be added to the list of known precipitating events.

\section{References}

1 Aho K. Pathogenesis of Reiter's syndrome and reactive arthritis. Scand J Rheumatol [Suppl] 1984; 52: 30-6.

2 Feldman C A. Staphylococcal scarlet fever. $N$ Engl J Med1962; 267: 877-8.

3 Chesney P J, David J P, Purdy W K, Wand P J, Chesney R W Clinical manifestations of the toxic shock syndrome. JAMAw 1981; 94: 156-63.

4 Todd J, Fishant M, Kapral F, Welch T. Toxic shock syndrom associated with phage group 1 staphylococci. Lancet 1978; ii: 1116-8.

5 Catterall $\mathrm{R}$ D. Clinical aspects of Reiter's disease. $\mathrm{Br}$ Rheumatol 1983; 22(suppl 2): 151-5. 